\title{
Resource Allocation for SWIPT Systems with Nonlinear Energy Harvesting Model
}

\author{
Yifan Hu, Mingang Liu, and Yizhi Feng \\ School of Electronic and Information Engineering, South China University of Technology, Guangzhou 510641, China \\ Correspondence should be addressed to Yizhi Feng; yzfeng@scut.edu.cn
}

Received 27 January 2021; Revised 5 March 2021; Accepted 19 March 2021; Published 7 April 2021

Academic Editor: Wei Duan

Copyright ( 2021 Yifan Hu et al. This is an open access article distributed under the Creative Commons Attribution License, which permits unrestricted use, distribution, and reproduction in any medium, provided the original work is properly cited.

\begin{abstract}
In this paper, we study the resource allocation for simultaneous wireless information and power transfer (SWIPT) systems with the nonlinear energy harvesting $(\mathrm{EH})$ model. A simple optimal resource allocation scheme based on the time slot switching is proposed to maximize the average achievable rate for the SWIPT systems. The optimal resource allocation is formulated as a nonconvex optimization problem, which is the combination of a series of nonconvex problems due to the binary feature of the time slotswitching ratio. The optimal problem is then solved by using the time-sharing strong duality theorem and Lagrange dual method. It is found that with the proposed optimal resource allocation scheme, the receiver should perform EH in the region of medium signal-to-noise ratio (SNR), whereas switching to information decoding (ID) is performed when the SNR is larger or smaller. The proposed resource allocation scheme is compared with the traditional time switching (TS) resource allocation scheme for the SWIPT systems with the nonlinear EH model. Numerical results show that the proposed resource allocation scheme significantly improves the system performance in energy efficiency.
\end{abstract}

\section{Introduction}

In traditional energy-constrained wireless networks, the wireless devices normally use batteries as energy source and require periodically recharging or replacing the batteries, which is difficult for a large number of wireless devices and even hazardous or impossible in some circumstances [1], resulting limited lifetime of the wireless devices and the networks.

Energy harvesting (EH) that allows the energy-limited wireless devices to harvest energy from the ambient environment is a promising solution for extending the lifetime of energy-constrained wireless networks. Among the EH technologies, simultaneous wireless information and power transfer (SWIPT) takes advantage of the radio frequency (RF) signal's ability of carrying both information and energy at the same time, providing great convenience of recharging to energy-constrained devices by harvesting energy from the RF signals. SWIPT is especially suitable for the wireless terminals with low-power consumption whereas hard to access.
The SWIPT technique has gained wide attention from both researchers and engineers since Varshney proposed the idea in 2008 [1]. In [2], the trade-off between the amount of the harvested energy and the achievable rate is studied for the SWIPT systems in the frequency selection channel with additive white Gaussian noise (AWGN). In [3], two kinds of SWIPT receivers, namely, time switching (TS) and power splitting (PS) receivers, are, respectively, proposed. Since they were proposed, the TS and PS receivers have attracted a lot of interest due to the simplicity of realization [4-8]. Specifically, the trade-off between the information and energy transmission is studied for the point-to-point single-input singleoutput (SISO) systems with a PS receiver in [4], which is extended to the point-to-point multiple-input single-output (MISO) systems with a TS receiver in [5]. In [6], the system throughput maximization is proposed for the MISO systems with TS and PS receivers. The TS and PS ratios are optimized to maximize the weighted sum rate of all receivers for the multiuser SISO orthogonal frequency-division multiplexing (OFDM) systems in [7]. Aiming at the minimization for the transmission power, the power allocation problem for 
the multiuser MISO downlink system is studied, and the optimal PS ratio is obtained in [8].

Besides the receiver design and the trade-off between the energy harvesting and information decoding, one of the other key issues in the implementation of SWIPT is efficient resource allocation [9-15]. In [9], the authors study the power and subcarrier allocation schemes for energyefficient SWIPT in multicarrier systems. In [10], the secrecy rate maximization is studied in an OFDM secrecy communication system. A multiuser OFDM system for maximizing the sum rate with a minimum transmit power constraint is designed in [11]. In [12], an optimal resource allocation policy is derived in a generalized WPCN where the devices can harvest energy from both multiple-antenna power station and ambient energy harvesting. In [13], the energy efficiency maximization is considered in large-scale multiple-antenna SWIPT systems. In [14], the authors propose an energy efficiency maximization optimization scheme for the multiuser multicarrier energy-constrained amplify-and-forward (AF) multirelay network. In [15], a robust energy-efficient optimization is designed for MIMO two-way relay networks with SWIPT.

Most of the aforementioned works about SWIPT systems consider the linear EH model, where the power conversion efficiency factor of the $\mathrm{EH}$ receiver is assumed to be a constant. However, it is found that the power conversion efficiency of the practical RF to direct current (DC) converter is affected by the input power, i.e., when the input power is greater than a certain threshold, the output power changes nonlinearly with the input power and shows a saturation characteristic [16]. Hence, the linear $\mathrm{EH}$ model cannot properly model the practical EH implementations and may lead to the resource mismatch in the resource allocation or the overestimation in the system performance evaluation [17]. In [16, 18], the parametric and logistic function-based nonlinear $\mathrm{EH}$ model and the piece-wise linear $\mathrm{EH}$ model are, respectively, proposed to capture the nonlinear saturation input-output characteristic of the practical EH circuit, which are further exploited for the various scenarios [19-23]. Specifically, in [19], the authors study the joint transmit power allocation and receive power splitting for SWIPT systems with the realistic nonlinear EH model. Considering a Nakagami- $m$ channel, the authors investigate the outage probability and reliable throughput of a multiuser wireless-powered SWIPT system in [20]. In [21], the analytical results on outage probability performance are presented for a cooperative relay-aid network with spectrum sensing and energy harvesting. In [22], the power split factor is optimized to minimize the outage probability for the AF relay system with PS receiver and the nonlinear $\mathrm{EH}$ model. In [23], considering imperfect channel state information (CSI) conditions, the authors analyse the outage probability for the multirelay SWIPT systems with PS receivers and the nonlinear $\mathrm{EH}$ model.

In this paper, we consider the single-input singleoutput (SISO) point-to-point SWIPT communication systems with TS receiver. The piece-wise linear $\mathrm{EH}$ model is considered to model the nonlinear saturation input- output characteristic for the $\mathrm{EH}$ circuit. We propose a simple optimal resource allocation scheme based on the time slot-switching strategy to maximize the average achievable rate for the systems. The information transmission block time $T$ is divided into $N$ time slots. Each time slot is used for information decoding (ID) or EH according to the optimal scheme. The optimal problem is formulated as a nonconvex optimization, which is first proved to satisfy the time-sharing condition and then solved by using the time-sharing strong duality theorem and Lagrange dual method.

Compared with work [24] where the linear EH model is considered, the major contribution of our work is that we consider the effect of the saturation characteristic of the practical nonlinear EH model and derive a more realistic optimal resource allocation scheme.

The rest of this paper is organized as follows. "System Model" introduces the system model. The optimal resource allocation design is proposed and the optimal problem is solved in "Design of Resource Allocation Optimization Algorithm." In "Numerical Results," the numerical results and discussion are presented. "Conclusions" concludes this paper.

\section{System Model}

We consider a SISO point-to-point SWIPT communication system as shown in Figure 1, where both the receiver $R_{x}$ and the transmitter $T_{x}$ have single antenna and $R_{x}$ is assumed to be energy-limited and could harvest energy from the received signals with a TS scheme. We assume that the channel between the transmitter and receiver is subjected to frequency flat and the block Rayleigh fading. The channel coefficient is denoted as $h$, which is a random variable following the complex Gaussian distribution with zero mean and variance $\sigma^{2}$. Without loss of generality, we assume that each time slot is normalized transmission time. In each time slot, the TS ratio $\beta$ is equal to 0 or 1 , indicating that the receiver implements $\mathrm{EH}$ or ID operations, respectively. The received signal can be expressed as

$$
y=\sqrt{\theta P} h x+n_{a}
$$

where $P$ is the transmit power, $x$ is the data symbol with unity power, i.e., $E\left[|x|^{2}\right]=1$ where $E[]$ means the mathematical expectation, $n_{a} \sim C N\left(0, \sigma^{2}\right)$ is the channel noise, $\theta=d^{-m}$ represents the path loss where $d$ is the distance between the source and the destination nodes, and $m$ represents the pathloss exponent. The achievable rate of the system based on the TS scheme can be expressed as

$$
R(\beta)=\beta \log _{2}\left(1+\frac{\theta P H}{\sigma^{2}}\right),
$$

where $\mathrm{H}=|h|^{2}$ is the channel power gain.

We use the piece-wise linear function to model the nonlinear saturation input-output characteristic of the $\mathrm{EH}$ 


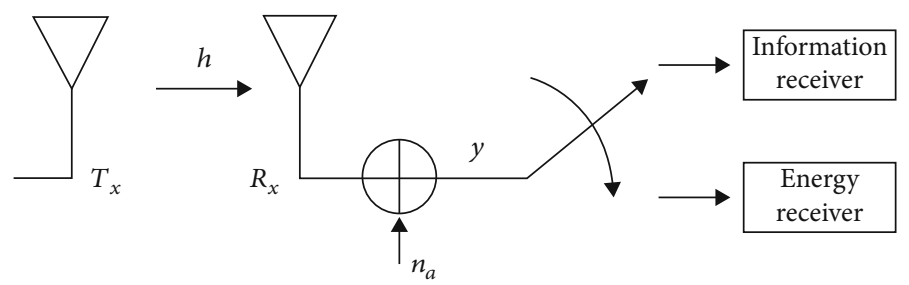

FIgUre 1: The SISO point-to-point SWIPT system.

receiver. The harvested power at the $\mathrm{EH}$ receiver is then given as $[17,18]$

$$
P_{o}=\left\{\begin{array}{l}
\eta \theta P H, \eta \theta P H<P_{s} \\
P_{s}, \eta \theta P H \geq P_{s}
\end{array}\right.
$$

where $\eta(0<\eta<1)$ is the energy conversion efficiency of the energy harvester in the linear region, $P_{s}$ is the maximum saturation harvested power of the EH receiver. As shown in (3), when the conversion power of the energy receiver $\eta \theta P H$ exceeds the saturation output power $P_{s}$, the output power of the energy receiver remains unchanged and some of the power is wasted, which means that in such case, the time slot should be switched to the ID receiver rather than $\mathrm{EH}$ receiver to avoid the waste of the power. Hence, the resource allocation scheme should be redesigned for the practical SWIPT system considering the nonlinear input-output characteristic of the EH circuit.

\section{Design of Resource Allocation Optimization Algorithm}

In this section, we propose an optimal resource allocation scheme based on the simple time slot-switching strategy to achieve the balance between the maximum average achievable rate and the maximum average harvested energy.

From (3), the harvested energy can be expressed as

$$
Q(\beta)=(1-\beta) P_{o}
$$

We consider maximizing the average achievable rate for the SISO SWIPT systems as shown in Figure 1. The optimization problem is formulated as

$$
\begin{array}{ll} 
& \max _{\beta} E[R(\beta)], \\
\text { s.t. } & E[Q(\beta)] \geq \bar{Q} \\
& \beta \in\{0,1\},
\end{array}
$$

where $\bar{Q}$ is the minimum amount of the harvested energy required to maintain the normal operation of the $\mathrm{EH}$ receiver.

In general, it is difficult to solve the optimal problem (5) directly since it is the combination of a series of nonconvex problems due to the binary feature of $\beta$. In addition, the complexity of solving the optimization problem using a numerical calculation method increases exponentially with the number of the time slots. In this section, to solve the optimization problem (5), we use the time-sharing strong duality theorem proposed in [25], which is given as follows.

We first prove that the optimization problem (5) satisfies the time-sharing condition (for more detail about the definition of time-sharing condition, the reader is referred to Ref. [25]).

Proposition 1. Let $\beta_{x}$ and $\beta_{y}$ be the optimal solutions of (5) with $\bar{Q}=Q_{x}$ and $\bar{Q}=Q_{y}$, respectively. Then, for any $\gamma \in[0,1]$ , there exists a feasible solution $\beta_{z}$ to the optimization problem (5) such that

$$
\begin{gathered}
E\left[R\left(\beta_{z}\right)\right] \geq \gamma E\left[R\left(\beta_{x}\right)\right]+(1-\gamma) E\left[R\left(\beta_{y}\right)\right], \\
E\left[Q\left(\beta_{z}\right)\right] \geq \gamma Q_{x}+(1-\gamma) Q_{y}
\end{gathered}
$$

and hence, the optimization problem (5) satisfies the timesharing condition.

Proof. Let $\beta_{x}$ be the feasible solution to the optimization problem (5).

(1) When $\gamma=0$, let $\beta_{z}=\beta_{y}$. Since $\beta_{y}$ is the optimal solution of the problem (5) when $\bar{Q}=Q_{y}$, the feasible solution $\beta_{z}$ obviously satisfies Equations (7) and (8)

(2) When $\gamma=1$, let $\beta_{z}=\beta_{x}$. Since $\beta_{x}$ is the optimal solution of the problem (5) when $\bar{Q}=Q_{x}$, the feasible solution $\beta_{z}$ also satisfies Equations (7) and (8)

(3) When $0<\gamma<1$, let $H_{k}$ denote the channel power gain of the $k \operatorname{th}(1 \leq k \leq N)$ time slot. The average achievable rate and the average harvested energy can be, respectively, expressed as

$$
\begin{aligned}
& E\left[R\left(\beta_{j}\right)\right]=\lim _{N \longrightarrow \infty} \frac{1}{N} \sum_{k=1}^{N} R_{k}\left(\beta_{j, k}\right), \\
& E\left[Q\left(\beta_{j}\right)\right]=\lim _{N \longrightarrow \infty} \frac{1}{N} \sum_{k=1}^{N} Q_{k}\left(\beta_{j, k}\right),
\end{aligned}
$$

where $j \in\{x, y, z\}, \beta_{j, k}$ is the value of $\beta_{j}$ in the $k$ th time slot. Define an integer $M$ such that $M=\lceil\gamma N\rceil$, where $\lceil\cdot\rceil$ means ceiling round operation. Let 


$$
\beta_{z, k}=\left\{\begin{array}{l}
\beta_{x, k}, k=1,2, \cdots, M \\
\beta_{y, k}, k=M+1, M+2, \cdots, N .
\end{array}\right.
$$

When $N \longrightarrow \infty$, it can be obtained that $M \longrightarrow \infty$ and $(N-M) \longrightarrow \infty$. Then, for any $\gamma \in(0,1)$, it can be derived that

$$
\begin{aligned}
E\left[R\left(\beta_{z}\right)\right] & =\lim _{N \longrightarrow \infty} \frac{1}{N} \sum_{k=1}^{N} R_{k}\left(\beta_{z, k}\right) \\
& =\lim _{N \longrightarrow \infty}\left[\frac{\gamma}{M} \sum_{k=1}^{M} R_{k}\left(\beta_{x, k}\right)+\frac{1-\gamma}{N-M} \sum_{k=M+1}^{N} R_{k}\left(\beta_{y, k}\right)\right], \\
& =\lim _{M \longrightarrow \infty} \frac{\gamma}{M} \sum_{k=1}^{M} R_{k}\left(\beta_{x, k}\right)+\lim _{N-M \longrightarrow \infty} \frac{1-\gamma}{N-M} \sum_{k=M+1}^{N} R_{k}\left(\beta_{y, k}\right) \\
& =\gamma E\left[R\left(\beta_{x}\right)\right]+(1-\gamma) E\left[R\left(\beta_{y}\right)\right],
\end{aligned}
$$

which means that the feasible solution $\beta_{z}$ satisfies the equation (7). Similarly, it can be derived that

$$
\begin{aligned}
\mathrm{E}\left[\mathrm{Q}\left(\beta_{\mathrm{z}}\right)\right] & =\lim _{\mathrm{N} \longrightarrow \infty} \frac{1}{\mathrm{~N}} \sum_{\mathrm{k}=1}^{\mathrm{N}} \mathrm{Q}_{\mathrm{k}}\left(\beta_{\mathrm{z}, \mathrm{k}}\right) \\
& =\lim _{\mathrm{N} \longrightarrow \infty}\left[\frac{\gamma}{\mathrm{M}} \sum_{\mathrm{k}=1}^{\mathrm{M}} \mathrm{Q}_{\mathrm{k}}\left(\beta_{\mathrm{x}, \mathrm{k}}\right)+\frac{1-\gamma}{\mathrm{N}-\mathrm{M}_{\mathrm{k}=\mathrm{M}+1}} \sum_{\mathrm{k}}^{\mathrm{N}} \mathrm{Q}_{\mathrm{y}, \mathrm{k}}\left(\beta_{\mathrm{k}}\right)\right] \\
& \left.=\lim _{\mathrm{M} \longrightarrow \infty} \frac{\gamma}{\mathrm{M}} \sum_{\mathrm{k}=1}^{\mathrm{M}} \mathrm{Q}_{\mathrm{k}}\left(\beta_{\mathrm{x}, \mathrm{k}}\right)+\lim _{\mathrm{N}-\mathrm{M} \longrightarrow \infty} \frac{1-\gamma}{\mathrm{N}-\mathrm{M}_{\mathrm{k}=\mathrm{M}+1}} \sum_{\mathrm{k}}^{\mathrm{N}} \mathrm{Q}_{\mathrm{y}, \mathrm{k}}\right), \\
& =\gamma \mathrm{E}\left[\mathrm{Q}\left(\beta_{\mathrm{x}}\right)\right]+(1-\gamma) \mathrm{E}\left[\mathrm{Q}\left(\beta_{\mathrm{y}}\right)\right] \geq \gamma \mathrm{Q}_{\mathrm{x}}+(1-\gamma) \mathrm{Q}_{\mathrm{y}}
\end{aligned}
$$

which means that the feasible solution $\beta_{z}$ satisfies Equation (8). From (11) and (12), Proposition 1 is proved.

Since the optimization problem (5) satisfies the timesharing condition, according to the Time-Sharing Strong Duality Theorem, the primal problem (5) has the same optimal solution as its dual problem and can be solved by the Lagrange dual method.

The Lagrange function of (5) can be expressed as

$$
L(\beta, \lambda)=E[R(\beta)]+\lambda(E[Q(\beta)]-Q),
$$

where $\lambda \geq 0$ is the Lagrange multiplier associated with $E[R($ $\beta)] \geq Q$. Accordingly, the Lagrange dual function can be expressed as

$$
g(\lambda)=\max _{\beta \in\{0,1\}} L(\beta, \lambda)
$$

The dual problem is then given as

$$
\begin{array}{ll}
\min & g(\lambda) \\
\text { s.t. } & \lambda \geq 0,
\end{array}
$$

In order to effectively solve the above dual problem, we first decouple the optimization problem (14) into parallel $N$ subproblems that has the same structure as (14). The $k$ th $(k=1,2, \cdots N)$ subproblem can be expressed as

$$
\max _{\beta \in\{0,1\}} L_{k}(\beta)
$$

where $L_{k}(\beta)=R(\beta)+\lambda Q(\beta)$. In order to solve the optimization problem (16), we compare the value of the objective function $L_{k}(\beta)$ when $\beta=1$ or $\beta=0$, which can be expressed as

$$
\begin{gathered}
L_{k}(\beta=1)=\log _{2}\left(1+\frac{\theta P H}{\sigma^{2}}\right), \\
L_{k}(\beta=0)=\lambda P_{o},
\end{gathered}
$$

respectively. Hence, in the optimal solution of problem (14), $\beta^{*}$ can be expressed as

$$
\beta^{*}=\left\{\begin{array}{l}
1, \log _{2}\left(1+\frac{\theta P H}{\sigma^{2}}\right)>\lambda P_{o} . \\
0, \text { else }
\end{array}\right.
$$

Then, for a given value $\lambda, \beta^{*}$ can be obtained from (18) according to the channel state in each time slot. Let $\lambda^{*}$ be the optimal dual variable, which is associated with the required minimum harvested energy value $\bar{Q}$ in the inequality constraint term in (5). The optimal dual variable $\lambda^{*}$ can be obtained by iterative search and updating until the average energy collection meets the minimum energy constraint, i.e., $E[Q(\beta)]=\bar{Q}$, for which the detailed iterative search algorithm will be discussed later.

The proposed resource allocation scheme is based on the optimal TS strategy according to the channel state in each time slot. To describe the optimal switching strategy (18) more clearly, from (3) and (18), we define two functions $G_{1}$ and $G_{2}$ with respect to the channel power gain

$$
\begin{aligned}
& G_{1}(H)=\log _{2}\left(1+\frac{\theta P H}{\sigma^{2}}\right)-\lambda^{*} \eta \theta P H, \\
& G_{2}(H)=\log _{2}\left(1+\frac{\theta P H}{\sigma^{2}}\right)-\lambda^{*} P_{s} .
\end{aligned}
$$

Equation (19) is not easy to solve because the first and second terms for $G_{1}(H)$ are, respectively, logarithmic and linear functions of $H$. In this paper, we solve it by traversing the value of $H$ from 0 , when the difference between the value of the first and second terms is close (we set it as $10^{-5}$ ), we consider the values of the two terms to be equal, i.e., $G_{1}(H)$ $=0$; then, we can obtain an approximate nonzero real root $H_{1}$. Next, it can be found by derivation that $G_{1}(H)$ is a function that increases first and then decreases, and the position of the point that changes its monotonicity is related to the value of $\lambda$. Obviously, we can find a $\lambda$ that takes the position in $\left(0, H_{1}\right)$. At this time, since $G_{1}(0)=0, G_{1}\left(H_{1}\right)=0$, 
when $H \in\left(0, H_{1}\right), G_{1}(H)>0$, from (3) and (18), $\log _{2}(1+(\theta$ $\left.\left.P H / \sigma^{2}\right)\right)>\lambda^{*} P_{0}=\lambda^{*} \eta \theta P H$, and hence, $\beta^{*}=1$. Similarly, it can be obtained by derivation that $G_{2}(H)$ is an increasing function, when $H \in\left(H_{2}, \infty\right), \log _{2}\left(1+\left(\theta P H / \sigma^{2}\right)\right)>\lambda^{*} P_{0}=$ $\lambda^{*} P_{s}, \beta^{*}=1$, where $H_{2}$ is the nonzero real root of equation $G_{2}(H)=0$. Then, it can be deduced that when $H \in\left[H_{1}, H_{2}\right]$, $\beta^{*}=0$. Therefore, the optimal TS strategy can be expressed as

$$
\beta^{*}(H)= \begin{cases}1, & H<H_{1} \text { or } H>H_{2} \\ 0, & H_{1} \leq H \leq H_{2} .\end{cases}
$$

In (21), the optimal TS thresholds $H_{1}$ and $H_{2}$ depend on the optimal dual variables $\lambda^{*}$, which is determined by the inequality constraints $\bar{Q}$ in (5) and should be chosen so that $E[Q(\beta(H))]=\bar{Q}$. The average energy collection can be expressed as

$$
E[Q(\beta(H))]=\int_{H_{1}}^{H_{t h}} \eta \theta P x f_{H}(x) d x+\int_{H_{t h}}^{H_{2}} P_{s} * f_{H}(x) d x,
$$

where $f_{H}(x)$ is the probability density function (pdf) of $H$, $H_{t h}=P_{s} / \eta \theta P$. The iterative search algorithm for the optimal dual variables $\lambda^{*}$ is summarized as follows in Algorithm 1. The initial value of $\lambda_{0}$ is set as 1.0 , and $\Delta \lambda$ is set as 0.01 .

The optimal resource allocation scheme can then be described as follows. Firstly, the information transmission block time $T$ is divided into $N$ time slots. Then, for each time slot, say, the $k \operatorname{th}(1 \leq k \leq N)$ time slot, the optimal TS thresholds $H_{1}$ and $H_{2}$ are determined by using Algorithm 1 . Finally, the channel power gain $H_{k}$ is compared with $H_{1}$ and $H_{2}$ in the $k \operatorname{th}(1 \leq k \leq N)$ time slot; if $H_{k}<H_{1}$ or $H_{k} \geq$ $\mathrm{H}_{2}$, the receiver switches to information decoding or else switches to energy harvesting. The optimal resource allocation scheme is summarized as follows in Algorithm 2.

Remark. It is shown in (21) and Algorithm 2 that for the SWIPT systems with nonlinear EH model, the optimal resource allocation scheme based on the time slot-switching strategy requires that the receiver switches to information decoding when the signal-to-noise ratio (SNR) is larger or smaller, whereas switching to energy harvesting is performed in the region of medium SNR.

\section{Numerical Results}

In this section, we present simulation results for the proposed optimal resource allocation scheme for the SWIPT systems with nonlinear EH model. In order to validate the proposed scheme, we compare the proposed resource allocation scheme with the traditional TS resource allocation scheme [26] in the energy efficiency performance for the SWIPT systems with nonlinear EH model, where the energy efficiency for the traditional TS scheme is obtained by sweeps, the time switching factor from 0 to 1 with a step 0.01 , and the energy efficiency is defined as

$$
\eta_{E}=R(\beta) /(P-E[Q(\beta)]) .
$$

Unless otherwise specified, the transmission power is set to $P=1 W$, and the required minimum harvested energy is set to $\bar{Q}=5 \mathrm{~mW}$. The energy conversion efficiency in the linear region and the saturation output power of the nonlinear $\mathrm{EH}$ receiver are set to $\eta=0.8$ and $P_{s}=24 \mathrm{~mW}$ [17], respectively. The variance of the additive white Gaussian noise is $\sigma^{2}=N_{0}$, and the SNR is defined as $\mathrm{SNR}=P / N_{0}$. The information transmission block time $T$ is divided into $N=10^{5}$ subslots, and in each subslot, the channel obeys the Rayleigh distribution and is independent of each other. The distance between the source node and the destination node is set to $d=5 \mathrm{~m}$, and the pathloss exponent $m$ is 2.0 .

Figure 2 shows the energy efficiency versus the transmit power $P$ for the SISO SWIPT systems with nonlinear EH model under various SNRs. It is shown that when the transmit power increases, the energy efficiency decreases in the region of middle and higher SNRs (i.e., $\mathrm{SNR}=15$ or $25 \mathrm{~dB}$ ), whereas it keeps almost invariant when the SNR is smaller, i.e., $\mathrm{SNR}=5 \mathrm{~dB}$. It can be observed that compared with traditional TS resource allocation scheme, the proposed resource allocation scheme significantly improves the system performance in energy efficiency. Furthermore, it can be also observed that the gap of system performance between the proposed scheme and traditional TS scheme becomes more obvious as the SNR increases, indicating that the proposed performs better in the region of higher SNR. The reason is that, when the SNR increases, the EH receiver is more likely to work in the nonlinear region. Due to the saturation characteristic of the nonlinear EH model, the traditional TS scheme is more likely to waste the received power in such case, thus resulting in the larger performance gap.

In Figure 3, the energy efficiency of the SISO SWIPT systems with nonlinear EH model under various SNRs is plotted against the distance $d$ between the source and the destination nodes. It can be observed that the energy efficiency decreases when the distance $d$ increases. It shows similar results in Figure 2 that the proposed resource allocation scheme substantially outperforms the traditional TS resource allocation scheme and that the performance gap gets larger when SNR increases. Moreover, it can be observed that as the distance $d$ increases, the energy efficiency of both schemes and the performance gap between the two schemes tend to be zero in the lower SNR region, since as the distance $d$ increases, the received power of the signal becomes very weak; thus, the achievable rate is very small in the lower SNR region for both the schemes.

Figure 4 shows the energy efficiency versus the pathloss exponent $m$ for the SISO SWIPT systems with the nonlinear EH model under various SNRs. Similar results can be observed in Figure 3 that the energy efficiency decreases when the pathloss exponent $m$ increases. The reason is that a larger value of $m$ means more transmit loss of the power of the signal, which brings a lower received SNR and smaller achievable rate for the system. It can be also observed that compared with the distance $d$, the system performance is more susceptible to the variation of the pathloss exponent $m$, since the pathloss exponent $m$ has a greater impact on pathloss than the source-destination distance $d$. Also, it is shown that the proposed resource allocation scheme substantially outperforms the traditional TS resource allocation 


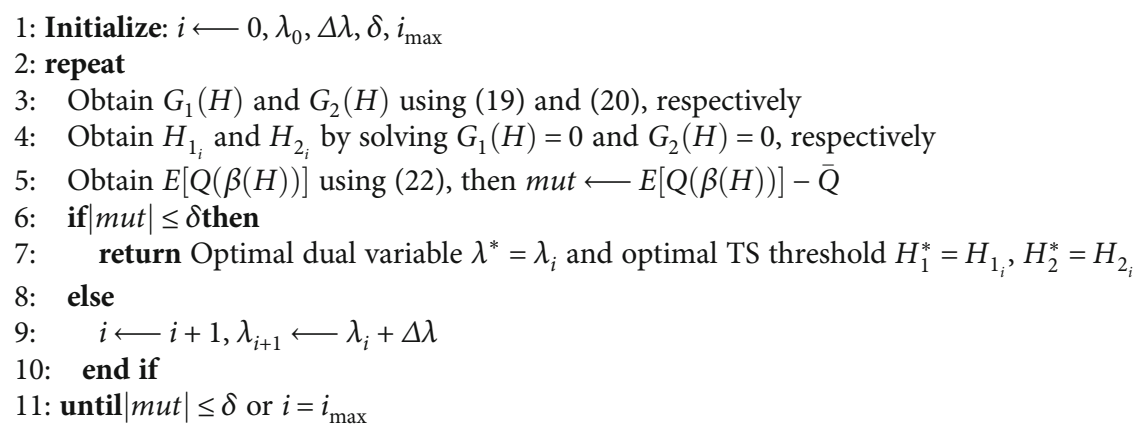

Algorithm 1: Iterative search algorithm for $\lambda^{*}$.

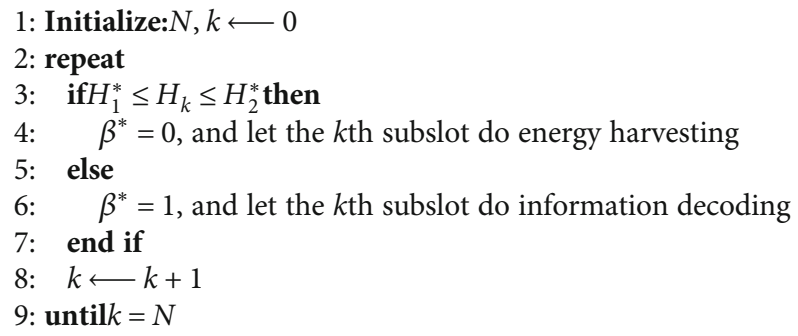

Algorithm 2: Resource allocation algorithm for the SWIPT systems with nonlinear EH model.

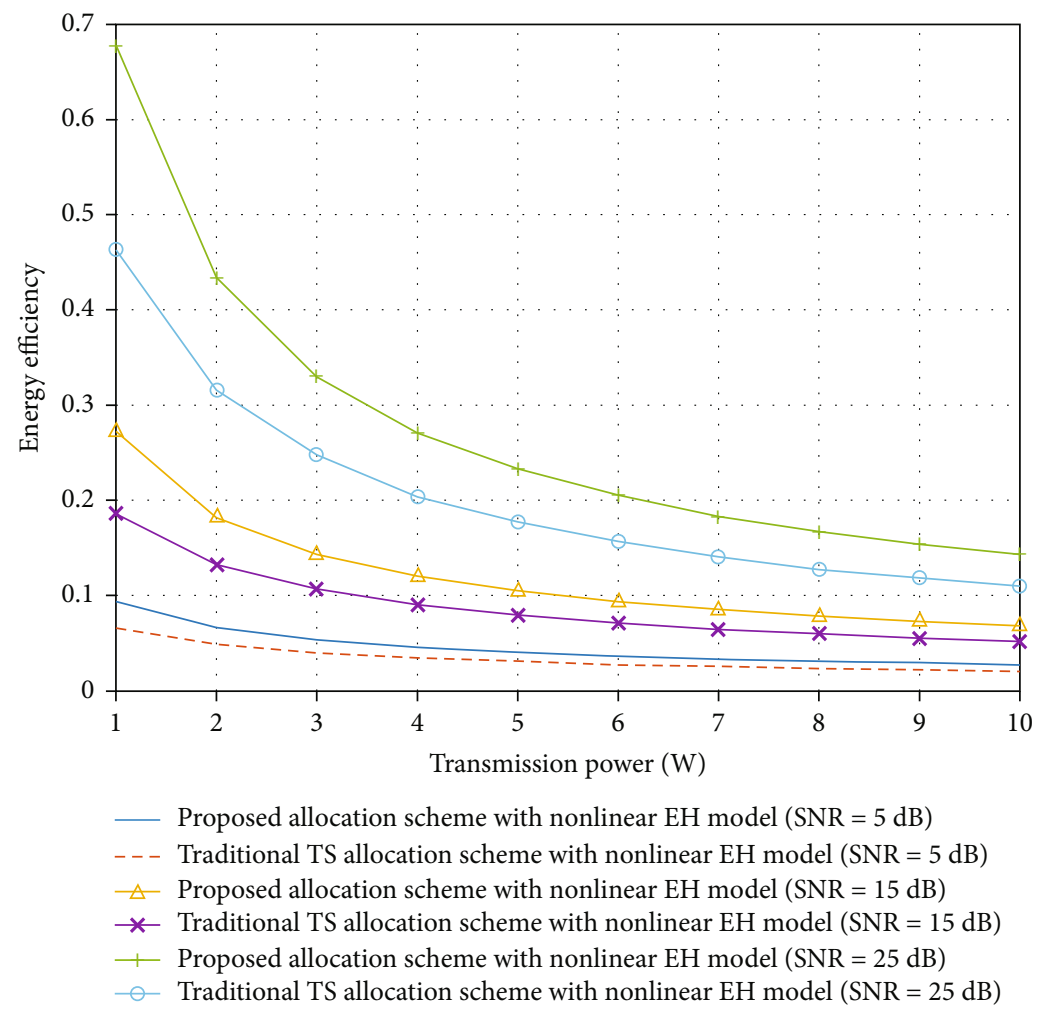

FIGURE 2: Energy efficiency versus transmission power for the SISO SWIPT systems with various signal-to-noise ratios (SNRs). 


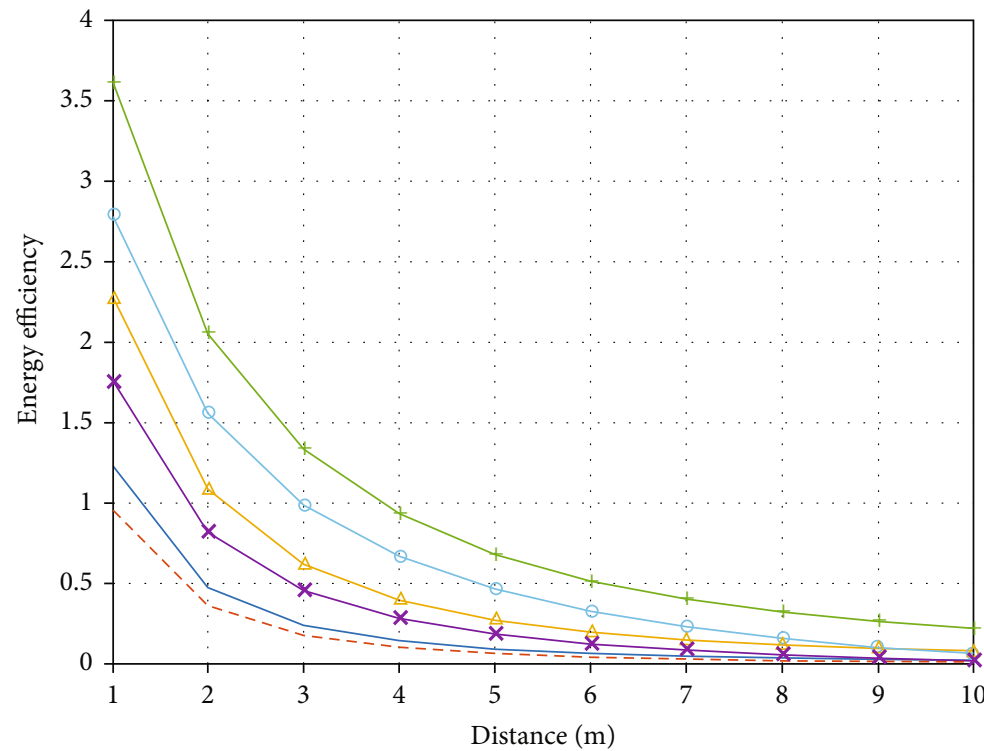

- Proposed allocation scheme with nonlinear EH model $(\mathrm{SNR}=5 \mathrm{~dB})$

- - - Traditional TS allocation scheme with nonlinear EH model $(\mathrm{SNR}=5 \mathrm{~dB})$

$\triangle$ Proposed allocation scheme with nonlinear EH model (SNR $=15 \mathrm{~dB})$

$*$ Traditional TS allocation scheme with nonlinear EH model $(\mathrm{SNR}=15 \mathrm{~dB})$

+ Proposed allocation scheme with nonlinear EH model $(\mathrm{SNR}=25 \mathrm{~dB})$

- Traditional TS allocation scheme with nonlinear EH model $(\mathrm{SNR}=25 \mathrm{~dB})$

FIGURE 3: Energy efficiency versus distance for the SISO SWIPT systems with various signal-to-noise ratios (SNRs).

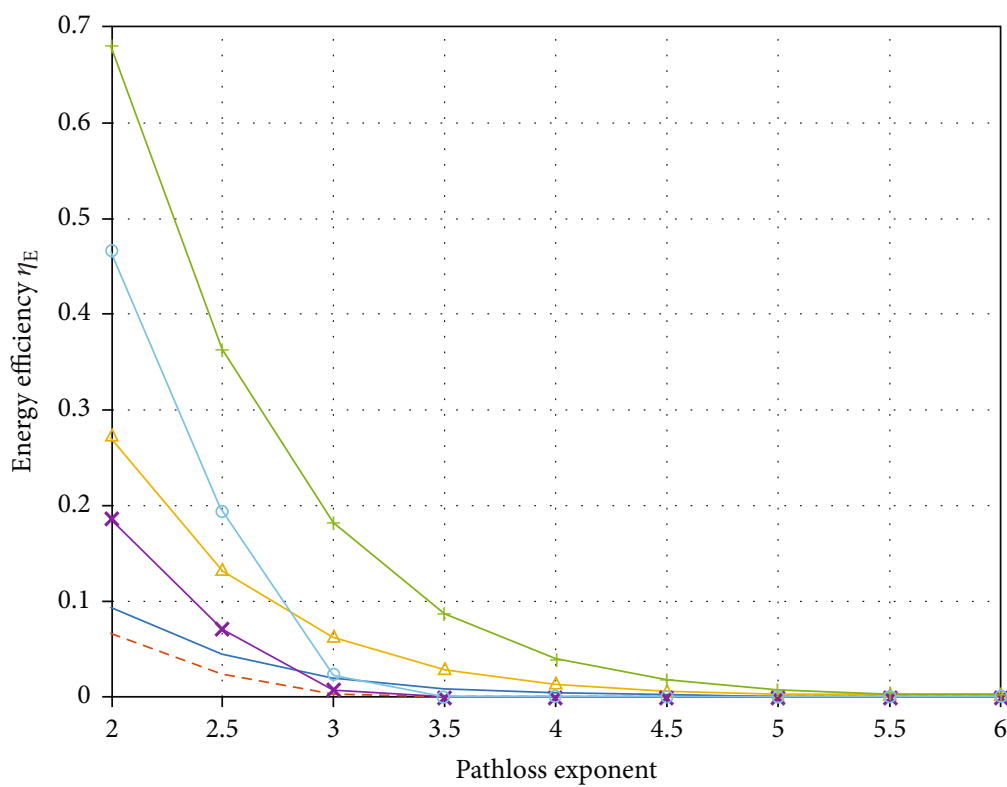

- Proposed allocation scheme with nonlinear EH model $(\mathrm{SNR}=5 \mathrm{~dB})$

- - - Traditional TS allocation scheme with nonlinear EH model $(\mathrm{SNR}=5 \mathrm{~dB})$

$\triangle$ Proposed allocation scheme with nonlinear EH model (SNR $=15 \mathrm{~dB})$

$*$ Traditional TS allocation scheme with nonlinear EH model $(\mathrm{SNR}=15 \mathrm{~dB})$

+ Proposed allocation scheme with nonlinear EH model $(\mathrm{SNR}=25 \mathrm{~dB})$

- Traditional TS allocation scheme with nonlinear EH model $(\mathrm{SNR}=25 \mathrm{~dB})$

FIGURE 4: Energy efficiency versus pathloss exponent for the SISO SWIPT systems with various signal-to-noise ratios (SNRs). 


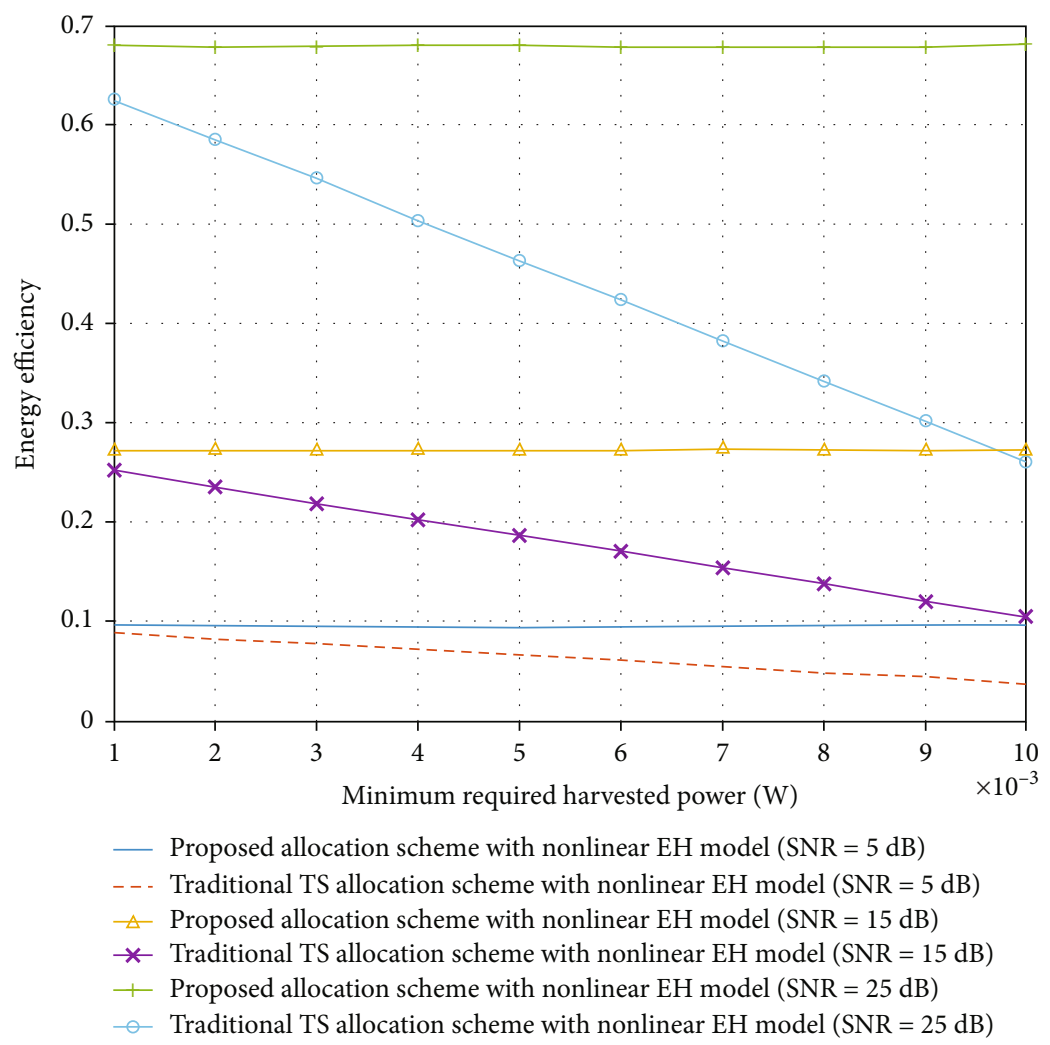

FIGURE 5: Energy efficiency versus minimum required harvested energy for the SISO SWIPT systems with various signal-to-noise ratios (SNRs).

scheme for smaller pathloss exponent $m$, whereas the energy efficiency of both schemes and the performance gap between the two schemes tend to be zero in lower SNR region when the pathloss exponent $m$ increases.

In Figure 5, the energy efficiency versus the minimum required harvested energy $\bar{Q}$ is shown for the SISO SWIPT systems with the nonlinear EH model under various SNRs. Not surprisingly, the proposed scheme substantially outperforms the traditional TS scheme despite the variation of the minimum required harvested energy. In fact, the energy efficiency of the system with the traditional TS scheme decreases with the increases of the minimum required harvested energy $\bar{Q}$, whereas for the proposed time slot-switching scheme, the system energy efficiency keeps almost unchangeable when the minimum required harvested energy $\bar{Q}$ increases from $1 \mathrm{~mW}$ to $10 \mathrm{~mW}$.

\section{Conclusions}

In this paper, we have studied the resource allocation scheme for the point-to-point SISO SWIPT systems with the nonlinear EH model. We have proposed an optimal resource allocation scheme based on the time slot switching to maximize the average information rate for the systems, with which the receiver performs information decoding in the region of higher or lower SNRs, whereas switching to energy harvesting is performed in the region of medium SNR. Compared to the traditional TS resource allocation scheme, the proposed scheme significantly improves the system performance in energy efficiency, and the system performance improvements gets larger when the SNR is higher. We have investigated the impacts of the source-destination distance $d$ and the pathloss exponent $m$ on the energy efficiency performance. Results have demonstrated that the system performance is more susceptible to the variation of the pathloss exponent $m$ than the distance $d$. We have also investigated the impacts of the minimum required harvested energy $\bar{Q}$ on the energy efficiency performance. It is demonstrated that for the traditional TS scheme, the system energy efficiency decreases with the increases of the minimum required harvested energy $\bar{Q}$, whereas for the proposed time slot-switching scheme, the system energy efficiency is hardly affected by the variation of the minimum required harvested energy $\bar{Q}$. In our setup, we consider the SISO SWIPT systems and Rayleigh channels for the proposed optimal scheme. MIMO systems and other more complex channel models for the proposed scheme can be further studied in future work.

\section{Data Availability}

The data used to support the findings of this study are available from the corresponding author upon request.

\section{Conflicts of Interest}

The authors declare that there is no conflict of interest regarding the publication of this paper. 


\section{Acknowledgments}

This research was funded by the National Natural Science Foundation of China (grant numbers 61871192 and 61871191) and the Science and Technology Program of Guangzhou (grant number 201904010373).

\section{References}

[1] L. R. Varshney, "Transporting information and energy simultaneously," in 2008 IEEE International Symposium on Information Theory, pp. 1612-1616, Toronto, ON, Canada, July 2008.

[2] P. Grover and A. Sahai, "Shannon meets Tesla: wireless information and power transfer," in 2010 IEEE International Symposium on Information Theory, pp. 2363-2367, Austin, TX, USA, June 2010.

[3] R. Zhang and C. K. Ho, "MIMO broadcasting for simultaneous wireless information and power transfer," IEEE Transactions on Wireless Communications, vol. 12, no. 5, pp. 1989-2001, 2013.

[4] L. Liu, R. Zhang, and K. C. Chua, "Wireless information and power transfer: a dynamic power splitting approach," IEEE Transactions on Communications, vol. 61, no. 9, pp. 39904001, 2013.

[5] H. Ju and R. Zhang, "A novel mode switching scheme utilizing random beamforming for opportunistic energy harvesting," IEEE Transactions on Wireless Communications, vol. 13, no. 4, pp. 2150-2162, 2014.

[6] C. Shen, W.-C. Li, and T.-H. Chang, "Wireless information and energy transfer in multi-antenna interference channel," IEEE Transactions on Signal Processing, vol. 62, no. 23, pp. 6249-6264, 2014.

[7] X. Zhou, R. Zhang, and C. K. Ho, "Wireless information and power transfer in multiuser OFDM systems," IEEE Transactions on Wireless Communications, vol. 13, no. 4, pp. 22822294, 2014.

[8] Q. Shi, L. Liu, W. Xu, and R. Zhang, "Joint transmit beamforming and receive power splitting for MISO SWIPT systems," IEEE Transactions on Wireless Communications, vol. 13, no. 6, pp. 3269-3280, 2014.

[9] D. W. K. Ng, E. S. Lo, and R. Schober, "Wireless information and power transfer: energy efficiency optimization in OFDMA systems," IEEE Transactions on Wireless Communications, vol. 12, no. 12, pp. 6352-6370, 2013.

[10] G. Zhang, J. Xu, Q. Wu, M. Cui, X. Li, and F. Lin, "Wireless powered cooperative jamming for secure OFDM system," IEEE Transactions on Vehicular Technology, vol. 67, no. 2, pp. 1331-1346, 2018.

[11] S. Yin and Z. Qu, "Resource allocation in multiuser OFDM systems with wireless information and power transfer," IEEE Communications Letters, vol. 20, no. 3, pp. 594-597, 2016.

[12] Q. Wu, G. Zhang, D. W. K. Ng, W. Chen, and R. Schober, "Generalized wireless-powered communications: when to activate wireless power transfer?," IEEE Transactions on Vehicular Technology, vol. 68, no. 8, pp. 8243-8248, 2019.

[13] X. Chen, X. Wang, and X. Chen, "Energy-efficient optimization for wireless information and power transfer in largescale MIMO systems employing energy beamforming," IEEE Wireless Communications Letters, vol. 2, no. 6, pp. 667-670, 2013.
[14] A. Gupta, K. Singh, and M. Sellathurai, “Time-switching EHbased joint relay selection and resource allocation algorithms for multi-user multi-carrier AF relay networks," IEEE Transactions on Green Communications and Networking, vol. 3, no. 2, pp. 505-522, 2019.

[15] Q. Li and L. Yang, "Robust optimization for energy efficiency in MIMO two-way relay networks with SWIPT," IEEE Systems Journal, vol. 14, no. 1, pp. 196-207, 2020.

[16] E. Boshkovska, D. W. K. Ng, N. Zlatanov, and R. Schober, "Practical non-linear energy harvesting model and resource allocation for SWIPT systems," IEEE Communications Letters, vol. 19, no. 12, pp. 2082-2085, 2015.

[17] Y. Feng, M. Wen, F. Ji, and V. C. M. Leung, "Performance analysis for BDPSK modulated SWIPT cooperative systems with nonlinear energy harvesting model," IEEE Access, vol. 6 , pp. 42373-42383, 2018.

[18] Y. Dong, M. J. Hossain, and J. Cheng, "Performance of wireless powered amplify and forward relaying over Nakagami- $m$ fading channels with nonlinear energy harvester," IEEE Communications Letters, vol. 20, no. 4, pp. 672-675, 2016.

[19] J. M. Kang, I. M. Kim, and D. I. Kim, “Joint Tx power allocation and Rx power splitting for SWIPT system with multiple nonlinear energy harvesting circuits," IEEE Wireless Communications Letters, vol. 8, no. 1, pp. 53-56, 2019.

[20] S. Gao, K. Xiong, R. Jiang, L. Zhou, and H. Tang, "Outage performance of wireless-powered SWIPT networks with nonlinear EH model in Nakagami-m fading," in 2018 14th IEEE International Conference on Signal Processing (ICSP), pp. 668-671, Beijing, China, August 2018.

[21] T. L. N. Nguyen and Y. Shin, "Outage probability analysis for SWIPT systems with nonlinear energy harvesting model," in 2019 International Conference on Information and Communication Technology Convergence (ICTC), pp. 196-199, Jeju Island, Korea (South), October 2019.

[22] K. Wang, Y. Li, Y. Ye, and H. Zhang, "Dynamic power splitting schemes for non-linear EH relaying networks: perfect and imperfect CSI," in 2017 IEEE 86th Vehicular Technology Conference (VTC-Fall), pp. 1-5, Toronto, ON, Canada, September 2017.

[23] J. Zhang and G. Pan, "Outage analysis of wireless-powered relaying MIMO systems with non-linear energy harvesters and imperfect CSI," IEEE Access, vol. 4, pp. 7046-7053, 2016.

[24] L. Liu, R. Zhang, and K. C. Chua, "Wireless information transfer with opportunistic energy harvesting," IEEE Transactions on Wireless Communications, vol. 12, no. 1, pp. 288-300, 2013.

[25] W. Yu and R. Lui, "Dual methods for nonconvex Spectrum optimization of multicarrier systems," IEEE Transactions on Communications, vol. 54, no. 7, pp. 1310-1322, 2006.

[26] X. Zhou, R. Zhang, and C. K. Ho, "Wireless information and power transfer: architecture design and rate-energy tradeoff," IEEE Transactions on Communications, vol. 61, no. 11, pp. 4754-4767, 2013. 